a Neanderthal. This means that to get the $1 \times$ coverage, or roughly 3 billion base pairs, of the genome, the team must sequence somewhere between 70 billion to 100 billion base pairs of these environmental samples.

As Egholm's team begins to comb through those environmental data contaminating the Neanderthal samples, he says the initial results have been surprising. "Whether these are recent bacteria, or ones that ate the poor guy when he died, we cannot be certain yet, but I can tell you from the first few contigs where we got multi-kilobase lengths - they matched nothing in GenBank."

\section{The next, next generation}

"The biggest change in metagenomics will come from 'third generation' sequencing systems or single-molecule sequencing," says Schuster. For the metagenomics community this nextnext-generation promises longer reads than Sanger sequencing, even higher throughput, lower costs and better quantitation of genes.

VisiGen Biotechnologies in Houston, Texas, is working on a method for sequencing single molecules in real-time that uses Förster resonance energy transfer (FRET). In this system, the polymerase is engineered to contain a donor fluorophore, and each nucleotide has a differently coloured acceptor fluorophore attached to the gamma

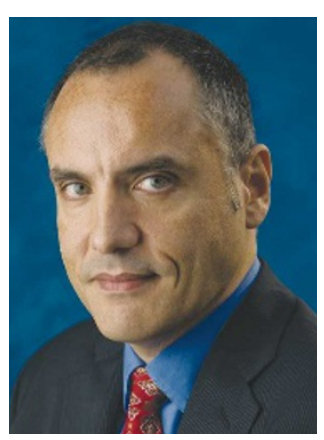

Isidore Rigoutsos is testing genome assembly. phosphate. When one of the nucleotides is incorporated by the polymerase into a native strand of DNA, a unique FRET signal is given off, and the pyrophosphate-contain-

ing fluorophore is then released leaving the synthesized strand of DNA ready for the next incorporation event. By imaging the colour changes as each incorporation occurs, VisiGen hopes to sequence single molecules in real-time and apply this on a massively parallel array with an output of up to one million bases per second.

Reveo in Hawthorne, New York, is developing a method to sequence DNA using nano-knifeedge probes, which pass over DNA that has been stretched and immobilized in a channel 10 micrometres wide. By using four different nano-knife-edge probes, each 'tuned' to a different frequency, Reveo hopes to non-destructively sequence DNA while eliminating the need for a costly imaging component. And Pacific Biosciences of Menlo Park, California, recently announced its single-molecule sequencing technology based on zero-mode waveguides ${ }^{2}$.
These new single-molecule sequencing methods show the promise of generating much longer read lengths in the future, says Schuster. VisiGen's method predicts sequence reads of 1,500 base pairs - the size of $16 \mathrm{~S}$ ribosomal RNA - whereas the Pacific Biosciences approach could produce reads as long as 10 kilobases.

And single-molecule sequencing is becoming a reality: in March 2008, Helicos BioSciences of Cambridge, Massachusetts, sold the first single-molecule sequencing system. The HeliScope uses a sequencing-by-synthesis approach in which the DNA is first fragmented into pieces $100-200$ base pairs long. Adaptors of known sequence are attached to the ends of the fragments so that they can be captured on

\title{
THE HUMAN ENVIRONMENT
}

"I don't know why the Human Microbiome Project bubbled to the top now instead of previously," says Jane Peterson from the National Institutes of Health (NIH). "Certainly there have been metagenomic studies done with the old technologies." But Peterson thinks recent reports exploring the human gut microbial community, along with new, advanced sequencing technologies, might have been enough to pique the interest of the reviewers who decided to fund the Human Microbiome Project (HMP).

The project is a 5-year, US\$115million effort to study the microbial communities inhabiting several regions of the human body, including the gastrointestinal and female urogenital tracts, oral cavity, nasal and pharyngeal tract, and skin, and how those communities influence human health and disease. The effort is viewed by many researchers in the metagenomics community as particularly timely as most agree improvements in sample collection standards and analysis tools are much needed. Karen Nelson from the J. Craig Venter Institute, who is also a participating investigator in the HMP, says these issues can finally be addressed with a project of this scope as all samples will collected and treated in the same way, and standards will be put in placefor annotation of the metagenomic data.

The project will fund research in several areas, although the construction of a data resource for sequencing DNA samples from as many as $\mathbf{2 5 0}$ individuals, and projects aimed at demonstrating how changes in the human microbiome are related to health and disease are centrepieces of the programme. Other project initiatives include the development of new metagenomics technology to isolate bacteria that are currently cannot be cultured, development of new bioinformatic programs and tools for analysis of large genomic data sets, data analysis and coordination centres, and analysing and understanding the ethical, legal and social issues of the project.

The HMP's initial sequencing efforts began this year. Peterson says that towards the end of the last fiscal year, the NIH Roadmap office had funds available for the HMP. Through existing relationships with large sequencing groups, the HMP was able to start quickly and generate preliminary sequencing data, which are being used for the other demonstration projects. This initial effort will result in the sequencing of $\mathbf{2 0 0}$ new bacterial organisms, recruitment of patients for metagenomic studies, and some $16 \mathrm{~S}$ ribosomal RNA metagenomic sequencing to assess microbial diversity at the various sites.

Peterson says that the protocols for sampling and recruitment have provided some early challenges. The HMP hopes to sample the same sites on all $\mathbf{2 5 0}$ individuals, but with so many sites, standardizing sampling can be tricky. "The protocol for the different sites has to be well worked out," she says, "the oral community has to be happy with the requirements that the skin community brings to the table."

Although it is just at the beginning, the HMP is scheduled to award its first rounds of grants to researchers this autumn. Peterson says that in the future the project's standardization efforts might not be restricted to the United States. "We are also forming an international consortium to coordinate international projects." 